The influence of boundary conditions on the accuracy of diffusion theory in time-resolved reflectance spectroscopy of biological tissues

This content has been downloaded from IOPscience. Please scroll down to see the full text. 1995 Phys. Med. Biol. 401957

(http://iopscience.iop.org/0031-9155/40/11/013)

View the table of contents for this issue, or go to the journal homepage for more

Download details:

IP Address: 131.215.70.231

This content was downloaded on 23/07/2016 at 00:32

Please note that terms and conditions apply. 


\title{
The influence of boundary conditions on the accuracy of diffusion theory in time-resolved reflectance spectroscopy of biological tissues
}

\author{
Andreas $\mathrm{H}$ Hielscher†ț, Steven L Jacquesț, Lihong Wang \\ Frank K Tittelt \\ $\dagger$ Department of Electrical and Computer Engineering, Rice University, 6100 South Main, \\ Houston, TX 77005-1892, USA \\ $\ddagger$ Laser Biology Research Laboratory, University of Texas MD Anderson Cancer Center, \\ 1515 Holcombe Boulevard, Houston, TX 77030, USA
}

Received 30 January 1995, in final form 30 June 1995

\begin{abstract}
The applicability of diffusion theory for the determination of tissue optical properties from time-resolved reflectance spectroscopy is investigated. Analytical expressions from diffusion theory using the three most commonly assumed boundary conditions at the air-tissue interface are compared with time-resolved Monte Carlo simulations and measurements on tissue phantoms. The effects of the choice of the boundary conditions on the accuracy of the findings for the optical parameters are quantified, and criteria for accurate curve-fitting algorithms are developed.
\end{abstract}

\section{Introduction}

The determination of optical properties (absorption $\mu_{a}$ and reduced scattering coefficient $\mu_{s}^{\prime}$ ) through time-resolved reflectance spectroscopy performed on the surface of biological tissues can yield useful information about a variety of physiological parameters (Wilson et al 1989). A few examples are blood-tissue oxygenation (Chance et al 1988, Benaron et al 1992), diabetes (Eppstein and Bursell 1992, Kohl et al 1995), diagnosis of cancer (Ramanujam et al 1994), and arteriosclerosis (Deckelbaum 1994). However, the correct quantitative interpretation of measured data sets with respect to the optical properties is still a major problem. Monte Carlo simulations have been found to describe the propagation of light in tissue with high accuracy (Flock et al 1989). The drawback of such simulations is that they are very time consuming and thus not desirable in a clinical environment. A much faster way to extract optical properties from experimental data is to fit analytical expressions derived from diffusion theory. However, diffusion theory has its weaknesses because it is only an approximation to the more accurate, but in general not analytically solvable, equation of radiative transfer. The complexity of the analytical expression found from diffusion theory varies furthermore with the assumed boundary conditions at the tissueair interface.

In the work presented here, the formulas found from diffusion theory under three commonly used boundary conditions (partial current, extrapolated, and zero) are first briefly introduced and compared with each other. It is shown that diffusion theory with the extrapolated boundary condition (EPB) and diffusion theory with the partial current boundary 
condition $(\mathrm{PCB})$ predict almost the same reflectance for a given set of optical properties and source-detector separation. Compared with these two cases, diffusion theory with the zero boundary condition (ZBC) underestimates the amplitude of the signal and displays sharper maxima.

The time-resolved reflectance determined by diffusion theory with its different boundary conditions is then compared with time-resolved Monte Carlo simulations. The errors in the determination of the optical properties $\left(\mu_{a}\right.$ and $\left.\mu_{s}^{\prime}\right)$ using fitting algorithms based on diffusion theory with the $\mathrm{ZBC}$ and $\mathrm{EPB}$ are quantified. It is shown that the absorption coefficient can be obtained with high accuracy, while both theories fail to give accurate values for $\mu_{s}^{\prime}$. Finally, Monte Carlo simulations are compared with time-resolved experiments on tissue phantoms.

\section{Diffusion theory}

\subsection{Basic equations}

The equation of radiative transfer (Case and Zweifel 1967, Ishimaru 1978b) forms the basis of almost all the modelling that is currently being conducted in tissue optics. However, in only the simplest cases is it possible to obtain an analytical solution to this equation. Applications to practical situations generally require restrictive approximations. Under the assumption that the reduced scattering coefficient, $\mu_{s}^{\prime}=(1-g) \mu_{s}$, where $\mu_{s}$ is the scattering coefficient and $g$ the mean cosine of the scattering angle, is much larger than the absorption coefficient, $\mu_{a}$, the diffusion equation for a homogenous medium can be derived (Patterson et al 1989, Ishimaru 1978a):

$$
(1 / c)(\partial / \partial t) \phi(r, t)-D \nabla^{2} \phi(r, t)+\mu_{a} \phi(r, t)=S(r, t)
$$

where $\phi(r, t)$ is the diffuse photon fluence rate at the position $r, c$ is the speed of light in the tissue, $S(r, t)$ describes the photon source, and $D=\left\{3\left[\mu_{a}+\mu_{s}^{\prime}\right]\right\}^{-1}$ is the diffusion coefficient.

In practical applications such as measurements on biological tissues, the photon fluence rate $\phi$ is not measured directly. The measurable quantity is the number of photons $R(r, t)$ that reach the surface per unit area per unit time at a given source-detector separation $r$. $R(r, t)$ is called the reflectance and can be calculated from Fick's law (Duderstadt and Hamilton 1976, Patterson et al 1989) by

$$
R(r, t)=|-D \nabla \phi(r, z, t)|_{z=0} \mid .
$$

Solutions for equation (1) have been given for a variety of geometries (Arridge et al 1992). In the study presented here, we concentrate on the solution for a semi-infinite medium and how this solution can be used to quantify the optical properties from a given data set. Before the different boundary conditions for the air-tissue interface are discussed, the source term $S(r, t)$ must be specified to solve the diffusion equation (1). This is equivalent to giving an initial condition. In practical applications the source is typically a laser beam which strikes the tissue at $90^{\circ}$. The points at which the first scattering events occur are distributed exponentially into the medium, and the source can be modelled as a line of isotropic point sources with different strengths. Usually, this source distribution is further simplified by assuming that all incident photons are initially scattered at a depth equal to the transport mean free path (Patterson et al 1989, Haskell et al 1994)

$$
z_{0}=\left[\mu_{a}+\mu_{s}^{\prime}\right]^{-1}
$$


so that the actual source term becomes a simple delta function

$$
S(r, t)=\delta\left(r=0, z=z_{0}, t=0\right) .
$$

In this geometry the air-tissue interface is located in the $a$ plane perpendicular to the $z$ axis, at $z=0$, and the laser beam propagates along the $z$ axis. The three most commonly applied boundary conditions that describe the air-tissue interface will now be briefly reviewed and compared.

\subsection{The partial current boundary condition $(P C B)$}

It is reasonable to assume that light leaving the scattering medium through an air-tissue interface does not return into the medium. This means that the photon flux from the air into the tissue has to be zero. For a semi-infinite volume containing scatterers for all $z>0$ the directional photon flux $J_{+z}$ at the surface is given in the diffusion approximation by (Glasstone 1955, Keijzer et al 1988, Moulton 1990, Haskell et al 1994)

$$
J_{+z}=\phi(r, z=0, t) / 4-\left.(A D / 2)(\partial / \partial z) \phi(r, z, t)\right|_{z=0}=0
$$

with

$$
A=\left(1+r_{d}\right) /\left(1-r_{d}\right)
$$

(see also figure 1). Here $r_{d}$ is the internal reflectance, due to a refractive index mismatch between the air and the tissue. Groenhuis et al (1983) offer an approximate expression for $r_{d}$ as a function of $n=n_{\text {tissue }} / n_{\text {air }}$ :

$$
r_{d}=-1.440 n^{-2}+0.710 n^{-1}+0.668+0.0636 n .
$$

The diffusion equation (1) with initial condition (4) and the so-called partial current boundary condition (5) can be solved analytically, for example with the Laplace transformation method (Carslaw and Jaeger 1959, Svaasand et al 1993). This leads to the following expression for the reflectance (Moulton 1990, Avraham et al 1991):

$$
R_{P C B}(r, t)=(4 D \pi c)^{-3 / 2} t^{-5 / 2} z_{0} \exp \left(-\mu_{a} c t\right) \exp \left(-\left(z_{0}^{2}+r^{2}\right) / 4 D c t\right) T_{P C B}(t)
$$

where

$$
T_{P C B}(t)=(1 / \alpha)\left(1-(1 / \sqrt{\alpha \beta}) \sqrt{\pi} \exp \left((1+\alpha)^{2} / \alpha \beta\right) \operatorname{erfc}((1+\alpha) / \sqrt{\alpha \beta})\right)
$$

with

$$
\begin{aligned}
& \alpha=z_{0} A / c t \\
& \beta=4 D A / z_{0}=4 A / 3
\end{aligned}
$$

and $\operatorname{erfc}(x)$ is the complementary error function defined by

$$
\operatorname{erfc}(x) \equiv \frac{2}{\sqrt{\pi}}\left(1-\int_{0}^{x} \exp \left(-\xi^{2}\right) \mathrm{d} \xi\right)
$$

Equation (8) can be fitted to a given data set to yield the optical parameters $\mu_{a}$ and $\mu_{s}^{\prime}$. Because $R_{P C B}$ depends highly nonlinearly on $\mu_{a}$ and $\mu_{s}^{\prime}$, a nonlinear fitting routine, for example the Levenberg-Marquardt algorithm (Press et al 1992), has to be applied. 


\subsection{The extrapolated boundary condition $(E P B)$}

Instead of solving equation (1) with the boundary condition (5) directly, often an alternative approach is pursued, invoking the principle of image sources (Glasstone 1955, Farrell et al 1992, Jacques et al 1993). If the photon fluence rate $\phi$ is extrapolated into the air, using a straight line with the same slope as at the air-tissue boundary, $\left.(\partial / \partial z) \phi(r, z, t)\right|_{z=0}$, the fluence rate vanishes at a distance $z_{e}$ (figure 1) given by

$$
z_{e}=\phi(z=0) /\left[\left.(\partial / \partial z) \phi(r, z, t)\right|_{z=0}\right]=2 A D \text {. }
$$

Here, use was made of equation (5). The distance $z_{e}$ is called the linear extrapolation distance and leads to the so-called extrapolated boundary condition

$$
\phi\left(r, z=-z_{e}, t\right)=0 \text {. }
$$

It is important to realize that, in postulating that the photon fiux vanishes at the extrapolated boundary, there is no implication, either from transport theory or diffusion theory, that the flux is actually zero there. The concept of the hypothetical boundary where the flux vanishes as a result of linear extrapolation is merely a convenient mathematical device used to obtain a simple boundary condition.

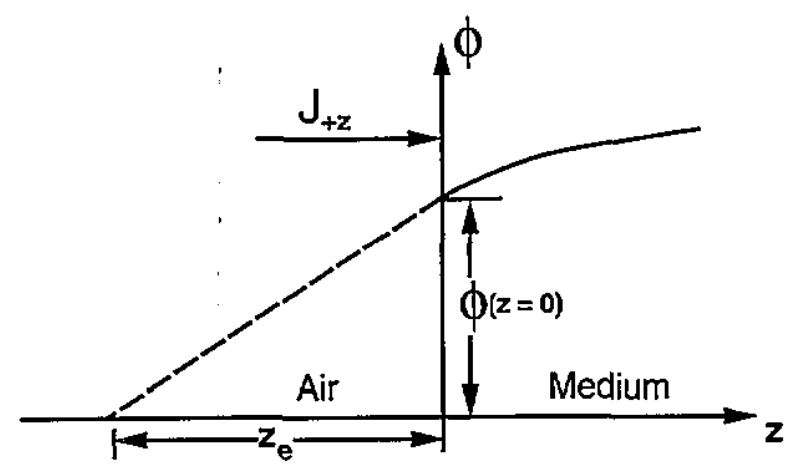

Figure 1. Extrapolation of the photon fluence at a plane interface between the diffusive medium and air.

The extrapolated boundary condition (14) is easily fulfilled if besides the original point source at $z_{0}$ an image source with the same strength but negative sign is placed at $z_{p}=z_{0}+2 z_{e}$ (figure 2) in an infinite medium. The time-dependent fluence, as discussed by Moulton (1990), can be written as a sum of two terms

$$
\begin{aligned}
\phi(r, z, t)=c & (4 D \pi c t)^{-3 / 2} \exp \left(-\mu_{a} c t\right) \\
& \times\left(\exp \left(-\left[\left(z-z_{0}\right)^{2}+r^{2}\right] / 4 D c t\right)-\exp \left(-\left[\left(z+z_{p}\right)^{2}+r^{2}\right] / 4 D c t\right)\right) .
\end{aligned}
$$

The first term is due to the point source inside the tissue at $z=z_{0}$ and the second term stems from the negative image source at $z=z_{\delta}$. Using equation (2), the reflectance becomes

$$
\begin{aligned}
R_{E P B}(r, t)= & \frac{1}{2}(4 D \pi c)^{-3 / 2} t^{-5 / 2} \exp \left(-\mu_{a} c t\right) \\
& \times\left(z_{0} \exp \left(-\left(z_{0}^{2}+r^{2}\right) / 4 D c t\right)+z_{p} \exp \left(-\left(z_{p}^{2}+r^{2}\right) / 4 D c t\right)\right) .
\end{aligned}
$$

Like $R_{P C B}, R_{E P B}$ depends nonlinearly on $\mu_{a}$ and $\mu_{s}^{\prime}$, and a nonlinear fitting routine has to be applied. However, the disappearance of the error function facilitates the fitting algorithm. The partial derivations with respect to $\mu_{a}$ and $\mu_{s}^{\prime}$, which are needed for the Levenberg-Marquardt routine, are readily found and are given in the appendix. 


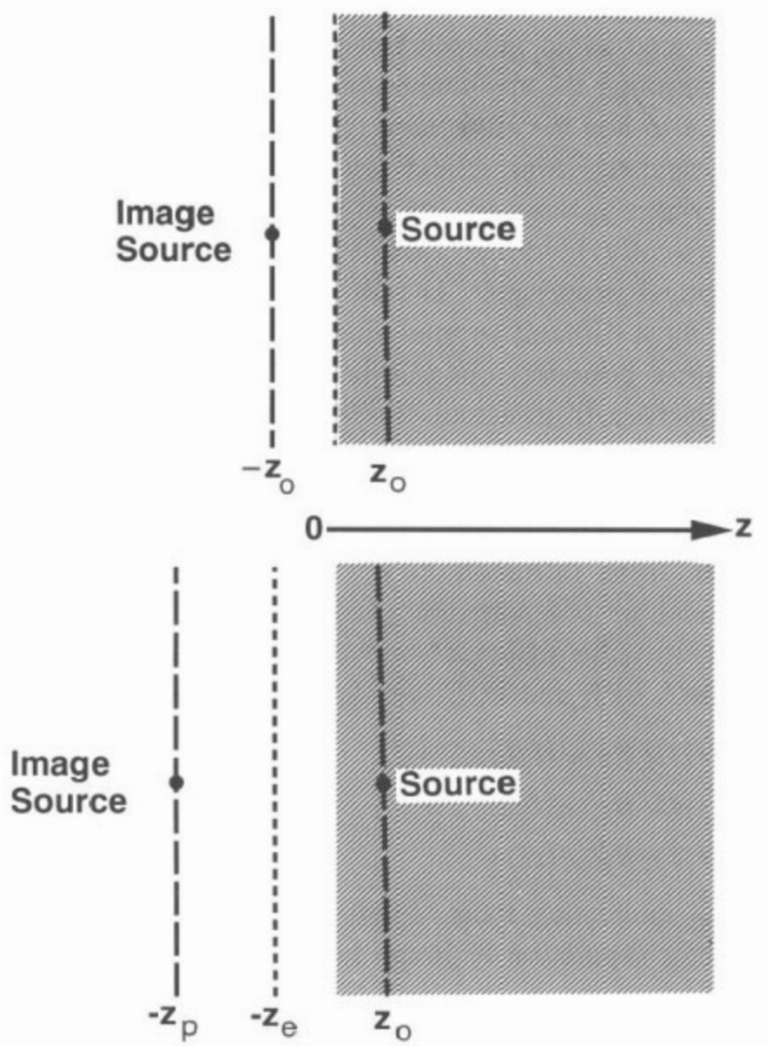

Figure 2. An illustration of the extrapolated and zcro-boundary conditions

\subsection{The zero-boundary condition $(Z B C$ )}

Physically incorrect but mathematically appealing is the so-called zero-boundary condition, which was first suggested by Patterson et al (1989). Here, the fluence $\phi$ is set to zero directly at the tissue-air interface rather than at an extrapolated distance $z_{e}$ above the surface. The boundary condition becomes

$$
\phi(r, z=0, t)=0
$$

and can be fulfilled by placing an image source with a negative sign at $z=-z_{0}$ (figure 2). The fluence is again a sum of two terms (Patterson et al 1989):

$$
\begin{aligned}
\phi(r, z, t)=c(4 D \pi c t)^{-3 / 2} \exp \left(-\mu_{a} c t\right) \\
\\
\quad \times\left(\exp \left(-\left[\left(z-z_{0}\right)^{2}+r^{2}\right] / 4 D c t\right)-\exp \left(-\left[\left(z+z_{0}\right)^{2}+r^{2}\right] / 4 D c t\right)\right) .
\end{aligned}
$$

Using equation (3), the reflectance is found:

$$
R_{Z B C}(r, t)=(4 D \pi c)^{-3 / 2} t^{-5 / 2} z_{0} \exp \left(-\mu_{a} c t\right) \exp \left(-\left(z_{0}^{2}+r^{2}\right) / 4 D c t\right) .
$$

To fit equation (19) to a given data set, it is advantageous to take the natural logarithm of both sides of the equation. This gives

$$
\ln \left[R_{Z B C}(r, t)\right]=\kappa-\frac{5}{2} \ln (t)-\left(c t+3 r^{2} / 4 c t\right) \mu_{a}-\left(3 r^{2} / 4 c t\right) \mu_{s}^{r}
$$

where $\kappa=\frac{3}{2} \ln \left(3\left(\mu_{\alpha}+\mu_{x}^{\prime}\right)\right)-\frac{3}{2} \ln (4 \pi c)+\ln \left(z_{0}\right)$. Furthermore, it is assumed that $r^{2} \gg z_{0}^{2}$, which holds true for most practical purposes. The $\mu_{s}^{\prime}$ and $\mu_{a}$ dependence of $\kappa$ should be 
ignored, and $\kappa$ should be treated as an independent fitting parameter because it is usually extremely problematic to determine the absolute amplitude of the reflectance signal in timeresolved experiments. This also has the advantage that equation (20) is linear with respect to the optical properties $\mu_{a}$ and $\mu_{s}^{\prime}$. Simple and fast mean least-squares fitting algorithms can be implemented to determine $\mu_{a}$ and $\mu_{s}^{\prime}$ from a given data set (Press et al 1992).

It appears that the physically incorrect $\mathrm{ZBC}$ yields the simplest mathematical tool for the determination of optical properties. The PCB, which is accurate in terms of the diffusion approximation, is most difficult to implement. In the following sections, we will establish which one of the three boundary conditions yields the most reliable theory for the determination of optical properties from actual measurements.

\subsection{Comparison of different boundary conditions}

Before we compare results from diffusion theory with Monte Carlo simulations and experiments, it is educational to compare the predictions of the theory with different boundary conditions. To quantity the difference between the prediction of the time-resolved diffusion theory with the ZBC, EPB and PCB, we define the ratios of

$$
\begin{aligned}
& R_{1}=R_{P C B}(r, t) / R_{Z B C}(r, t) \\
& R_{2}=R_{P C B}(r, t) / R_{E P B}(r, t) \\
& R_{3}=R_{E P B}(r, t) / R_{Z B C}(r, t) .
\end{aligned}
$$

One can observe several interesting facts. First, the ratios only depend on the time $t$ and not on the source-detector separation $r$. It can be easily seen that

$$
R_{1}=T_{P C B}(t)
$$

and

$$
R_{3}=\frac{1}{2}\left(1+\left(z_{p} / z_{0}\right) \exp \left(\left(z_{0}^{2}-z_{p}^{2}\right) / 4 D c t\right)\right)
$$

From this follows

$$
R_{2}=R_{1} / R_{3}=2 T_{P C B}(t)\left(1+\left(z_{p} / z_{0}\right) \exp \left(\left(z_{0}^{2}-z_{p}^{2}\right) / 4 D c t\right)\right)^{-1}
$$

Furthermore, for the limit $t \rightarrow \infty$, we find that

$$
R_{3}(t \rightarrow \infty)=R_{1}(t \rightarrow \infty)=1+\frac{2}{3} A \equiv \eta
$$

which leads to

$$
R_{2}(t \rightarrow \infty)=1
$$

$R_{3}(t \rightarrow \infty)$ can be determined from equation (23) by expanding the exponent and using $z_{p}=z_{0}+2 z_{e}=z_{0}+4 A D$ and $D / z_{0}=\frac{1}{3}$. To derive $R_{1}(t \rightarrow \infty)$, first use has to be made of the asymptotic expansion of the complementary error function (Abramowitz and Stegun 1972):

$$
\operatorname{erfc}(x \rightarrow \infty) \sim\left[\exp \left(-x^{2}\right) / \sqrt{\pi}\right]\left(1 / x-1 / 2 x^{3}+\mathrm{O}\left(1 / x^{5}\right)\right)
$$

with $x=(1+\alpha) / \sqrt{\alpha \beta}$ (see equations (8)-(12)). Subsequently, the binomial expansion has to be applied to the terms $(1+\alpha)^{-n}$. Equation (25) shows that at late times, the ratios $R_{1}$ and $R_{3}$ depend only on the index mismatch of the tissue-air interface and that $R_{P C B}$ and $R_{E P B}$ are the same for $t \rightarrow \infty$. Assuming a value of $n_{\text {tissue }}=1.35$, we obtain from equations (7) and (6), $A=2.919$ and subsequently from equation (25), $\eta=2.946$. This means that $R_{Z B C}$ underestimates the reflectance by a factor of 2.946 for $t \rightarrow \infty$. Furthermore, equations (23) and (24) suggest that the influence of $\mu_{a}$ on the ratios defined above is small, while a rather 

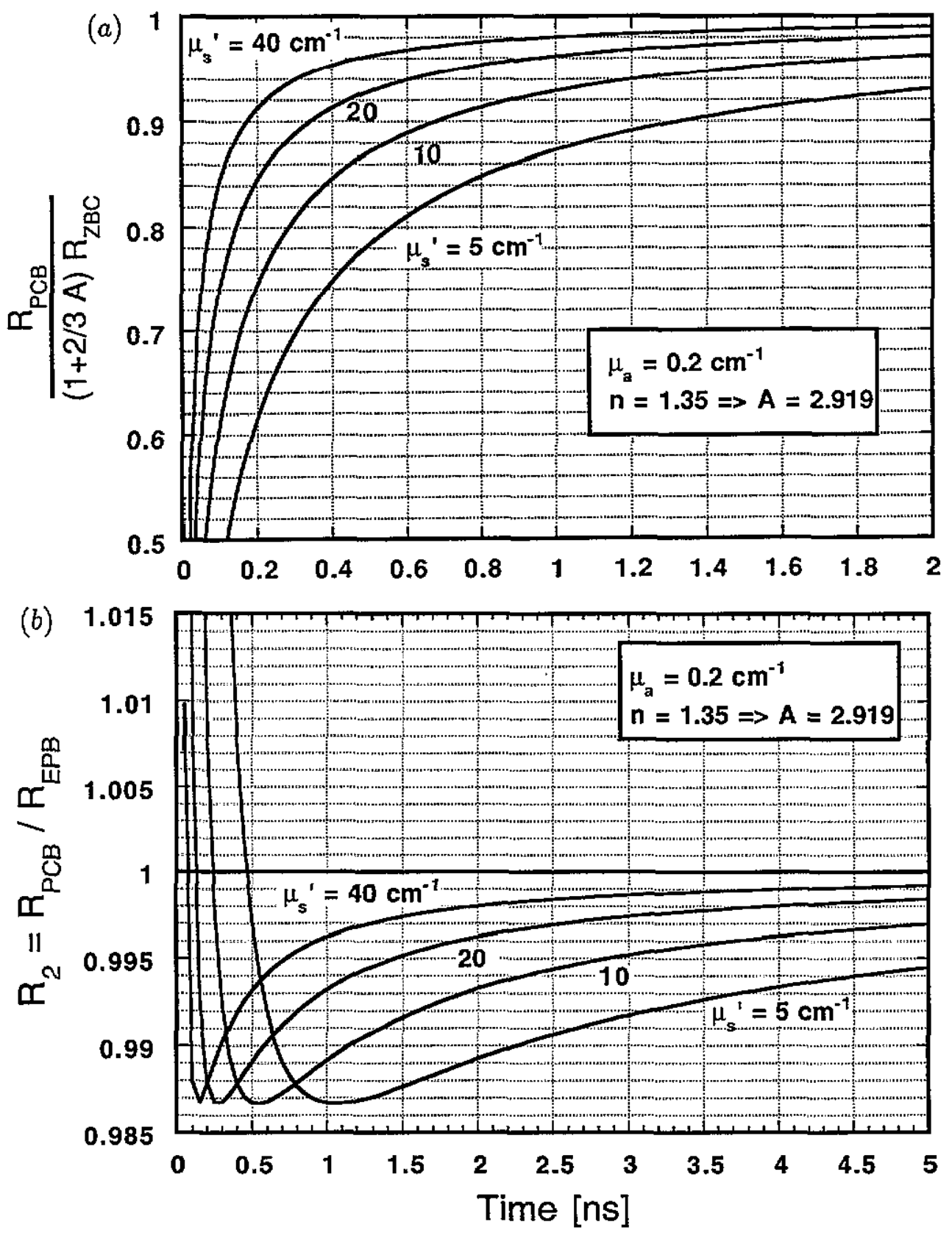

Figure 3. The ratio of reflectance calculated with $R_{P C B}$ and (a) $[1+2 / 3 A] R_{Z B C}$ and (b) $R_{E P B}$ as a function of time, for various $\mu_{s}^{\prime}$.

strong dependence on $\mu_{s}^{\prime}$ can be observed. Figure 3(a) displays the ratio of $R_{P C B}$ divided by $\eta R_{Z B C}$ as a function of $t$ for different $\mu_{s}^{\prime}$. The absorption coefficient $\mu_{a}$ is $0.2 \mathrm{~cm}^{-1}$ in all cases. In general, it can be seen that for small $t, \eta R_{Z B C}$ overestimates the signal. As $t$ increases the difference between $R_{P C B}$ and $\eta R_{Z B C}$ becomes smaller and smaller. The two theories merge faster as $\mu_{s}^{\prime}$ is increased. To obtain an error smaller than $10 \%, \sim 1.3 \mathrm{~ns}$ has 
to elapse for the case of $\mu_{s}^{\prime}=5 \mathrm{~cm}^{-1}$, but only $160 \mathrm{ps}$ have to pass for $\mu_{s}^{\prime}=40 \mathrm{~cm}^{-1}$.

Figure 3(b) displays the ratio $R_{2}$, that is the predicted reflectance when the PCB is used divided by the predicted reflectance when the EPB is used. It is shown that for $t \rightarrow \infty$ the ratio $R_{2}$ is equal to unity and no differences between the two boundary conditions exist (equation (26)). For small times, $R_{2}$ is larger than unity which means that $R_{E P B}$ yields a too small signal. For large times, $R_{2}$ is smaller than unity, therefore $R_{E P B}$ is slightly larger than $R_{P C B}$. However the difference between $R_{E P B}$ and $R_{P C B}$ is very small and almost always less than $2 \%$. The two theories agree better as $\mu_{s}^{\prime}$ increases.

\section{Monte Carlo simulations and diffusion theory}

\subsection{Monte Carlo simulations}

Monte Carlo simulations are one of the most powerful tools available for the solution of radiative transfer problems. The advantage of Monte Carlo simulations is that no approximations are necessary, and complex geometries and optical inhomogeneities can be modelled. As a result, Monte Carlo simulations have been used extensively to study a variety of transport problems, especially in reactor theory (Briesmeister 1986, Lux and Koblinger 1991). In the study presented here, Monte Carlo simulations were used as 'gold standards' to test the results of the diffusion theory calculations.

A steady state Monte Carlo model (Wang et al 1995) was adapted to simulate timeresolved propagation of photons in a semi-infinite medium (Jacques et al 1995). A $\delta$ function pulse is injected into the tissue with given $\mu_{a}, \mu_{s}$, and $g$. The reflectance $R(r, t)$ in $\mathrm{mm}^{-2} \mathrm{~ns}^{-1}$ is recorded as a function of the source-detector separation $r$ and time $t$. The spatial resolution was $1 \mathrm{~mm}$ over a total distance of $4 \mathrm{~cm}$. The time resolution was chosen to be $10 \mathrm{ps}$ over a total time of $2.56 \mathrm{~ns}$, giving 256 data points. The Monte Carlo model accounts for the index mismatch at the air-tissue boundary by calculating the Fresnel reflection (Wang et al 1995).

For most of the simulations in this work, two million photons were launched into the tissue. Each simulation took $\sim 12-48 \mathrm{~h}$ on a Sun SPARC station 10, depending on the optical properties. We found that the larger the ratio $\mu_{s}^{\prime} / \mu_{a}$, the longer the time for one simulation (Wang and Jacques 1995). Acceptable standard deviations for each data point could be achieved for source-detector separation up to $4 \mathrm{~cm}$ when $\mu_{s}^{\prime} / \mu_{a}<30$. With increasing $\mu_{s}^{\prime} / \mu_{a}$ ratio the source-detector separation had to be decreased to assure accurate Monte Carlo simulation results in reasonable computation time. To further decrease highfrequency noise a moving average algorithm (Press et al 1992) was applied to smooth the data.

\subsection{Comparison of Monte Carlo simulations with diffusion theory with the $Z B C$}

Figure 4(a) shows Monte Carlo simulations for a medium with $\mu_{a}=0.2 \mathrm{~cm}^{-1}, \mu_{s}^{\prime}=5 \mathrm{~cm}^{-1}$, and $g=0.92$, for different source-detector separations. Also displayed are the reflectance $\eta R_{Z B C}$ predicted by diffusion theory with the $\mathrm{ZBC}$ (equation (19)) and the reflectance $R_{E P B}$ predicted by diffusion theory with the EPB (equation (16)). The error bars indicate selected standard deviations that were calculated from 10 simulations with the same optical properties and source-detector separations. In this section only the $\eta R_{Z B C}$ curves are compared to the Monte Carlo simulations. The $R_{E P B}$ curves will be discussed in the next section. As can be seen, for times larger than $\sim 1 \mathrm{~ns}$ diffusion theory and Monte Carlo simulations agree very well. For smaller times, $\eta R_{Z B C}$ increasingly overestimates the reflectance. The largest 

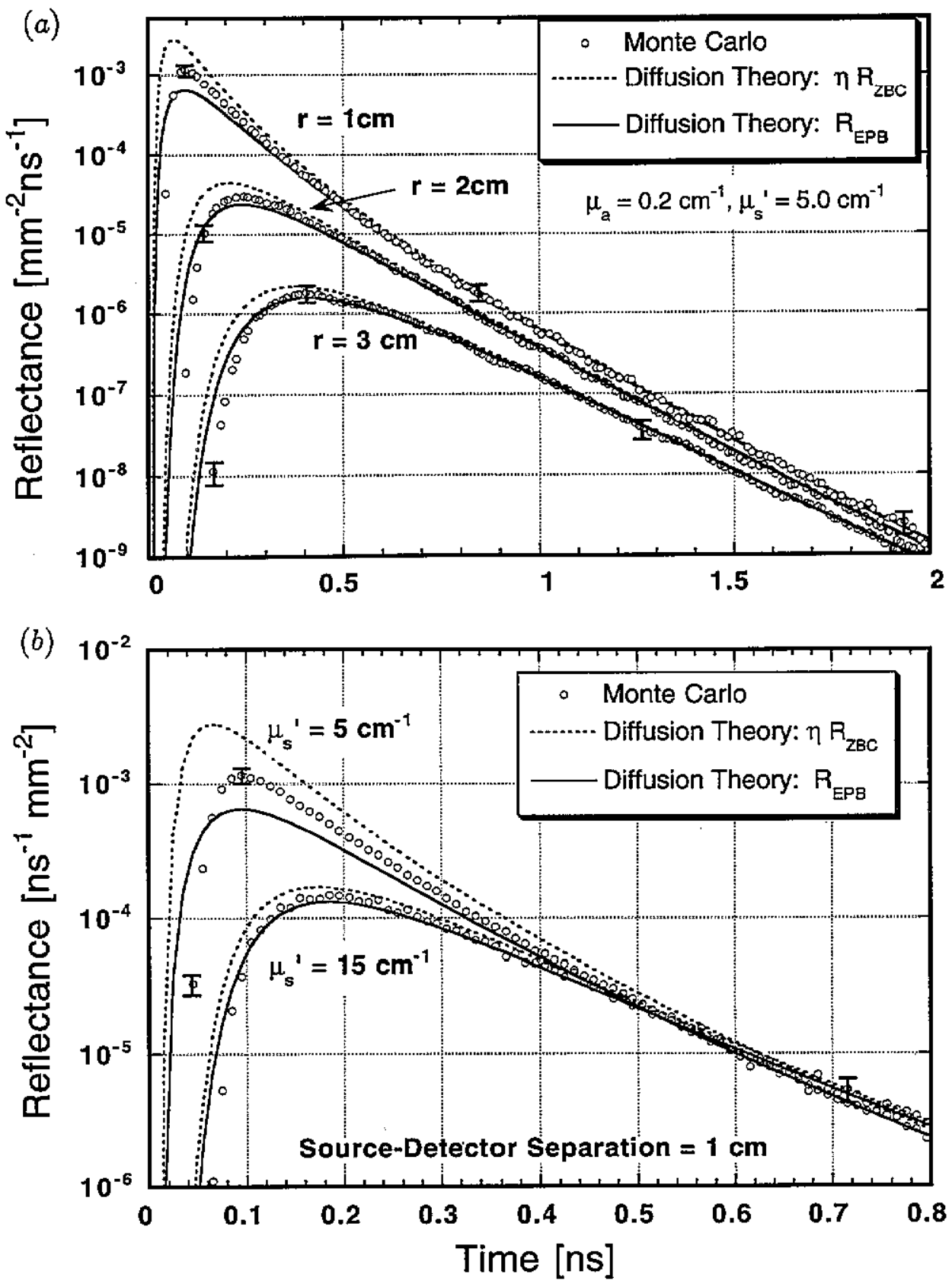

Figure 4. A comparison of Monte Carlo simulations with reflectance predicted by $\eta R_{Z B C}$ and $R_{E P B}$ for (a) three different source-detector separations, where the optical properties are the same in all cases $\left(\mu_{a}=0.2 \mathrm{~cm}^{-1}\right.$ and $\mu_{s}^{\prime}=5 \mathrm{~cm}^{-1}$ ) and (b) two different $\mu_{s}^{\prime}$, where the absorption coefficient is $\mu_{a}=0.2 \mathrm{~cm}^{-1}$, and the source-detector separation is $1 \mathrm{~cm}$.

differences can be observed for the smallest source-detector separations of $1 \mathrm{~cm}$.

Figure 4(b) displays Monte Carlo simulations and predictions of $\eta R_{Z B C}$ and $R_{E P B}$ for two different reduced scattering coefficients. The source-detector separation is fixed at $1 \mathrm{~cm}$. As the scattering coefficient increases, agreement between simulations and diffusion theory 
improves. Thus, increasing either the source-detector separation or the reduced scattering coefficient leads to a better agreement between diffusion theory and simulations, similar to findings reported by Jacques (1989).

To quantify the agreement between diffusion theory with the ZBC and Monte Carlo simulations, a linear least-squares fitting algorithm based on equation (20) is used to extract $\mu_{a}$ and $\mu_{s}^{\prime}$ from the Monte Carlo simulations displayed in figure 4. For the fit the data points are weighted by the inverse of their variance. However, not all data available from the simulations are used for the fit. The typical dynamic range of time-resolved experiments based on time-correlated single-photon counting is $10^{4}$. Therefore, only data points with a reflectance of at least $10^{-4}$ times the maximum reflectance $R_{\max }\left(t_{\max }\right)$ are used for the fit. This defines a time range $\left[t_{a}, t_{b}\right]$ from which data points are taken. Furthermore, a variable offset time $t^{*}$ from $t_{a}$ is introduced, before which all data points are disregarded for the fit. This is done to investigate how neglecting various portions of the initial slope influences the outcome of the fit. It has just been shown (figure 4) that the discrepancy between diffusion theory and Monte Carlo simulation is largest for small $t$. Increasing $t^{*}$ means cutting increasing amounts of the early part of the reflectance. The fitting algorithm yields different values for $\mu_{s}^{\prime}$ (fit) and $\mu_{a}$ (fit) depending on $t^{*}$. These values are compared with the known optical properties from the Monte Carlo simulations, $\mu_{s}^{\prime}(\mathrm{MC})$ and $\mu_{a}(\mathrm{MC})$, by defining the relative fitting errors for $\mu_{a}$ or $\mu_{s}^{\prime}$ (denoted $\mu_{a l s}^{\prime}$ ) as

$$
\text { relative } \mu_{\text {als }}^{\prime} \text { fitting error }=\left[\mu_{\text {als }}^{\prime}(\mathrm{fit})-\mu_{\text {als }}^{\prime}(\mathrm{MC})\right] / \mu_{\text {als }}^{\prime}(\mathrm{MC}) \text {. }
$$

Figure 5(a) displays the relative error in the determination of $\mu_{s}^{\prime}$ as a function of the above-defined offset time $t^{*}$ for three different source-detector separations. The optical properties are the same for all three curves. The arrows indicate $t^{*}$ for which only data points with $t \geqslant t_{\max }$ are admitted for the fit. This means that all data points before the maximum reflection $R_{\max }$ are disregarded. Taking all data leads to a strong overestimation of $\mu_{s}^{\prime}$ in all cases. This overestimation is reduced when $t^{*}$ is increased, which means more of the leading edge of the impulse response is disregarded for the fit. For a source-detector separation of $1 \mathrm{~cm}$, the minimal error occurs when the data are fitted for $t \geqslant t_{\max }$. However, even in this case, $\mu_{s}^{\prime}$ is still overestimated by $25 \%$. An increase in source-detector separation leads to a smaller error in the $\mu_{s}^{\prime}$ determination. If the source-detector separation becomes larger than $3 \mathrm{~cm}$ and the data are fitted for points $t \geqslant t_{\max }$, the error in the determination of $\mu_{s}^{\prime}$ becomes smaller than $5 \%$.

In figure $5(\mathrm{~b})$, the $\mu_{s}^{\prime}$ fitting error is shown for three different scattering coefficients while the source-detector separation is kept constant. With increasing scattering coefficient the determination of $\mu_{s}^{\prime}$ becomes more accurate. The largest error occurs when all data points are used for the fit. Increasing $t^{*}$ leads to a smaller error. However, omitting too many points beyond $t_{\max }$ results in a larger variance of the fitting parameters because the number of points available for a fit decreases. We found that fitting data for points $t \geqslant t_{\max }$ gives the best results in terms of accuracy and smallest variance in the extraction of $\mu_{s}^{\prime}$. Figure 6 shows the findings for the determination of $\mu_{a}$. Again, using all data leads to an overestimation of $\mu_{a}$; here, however, only by $6-14 \%$. Omitting some early data points gives excellent fits of $\mu_{a}$ even for small source-detector separations and low scattering coefficients.

We conclude that with a simple linear fitting algorithm based on $R_{Z B C}(r, t)$, that is the time-resolved reflection determined by diffusion theory with the $\mathrm{ZBC}$, it is possible to determine the reduced scattering coefficient within 5\% accuracy when the source-detector separation is larger than $3 \mathrm{~cm}$ or $\mu_{s}^{\prime} / \mu_{a}>50$. The determination of $\mu_{a}$ can be considered exact within $2 \%$ as long as very early parts of the time-resolved reflectance are neglected. 


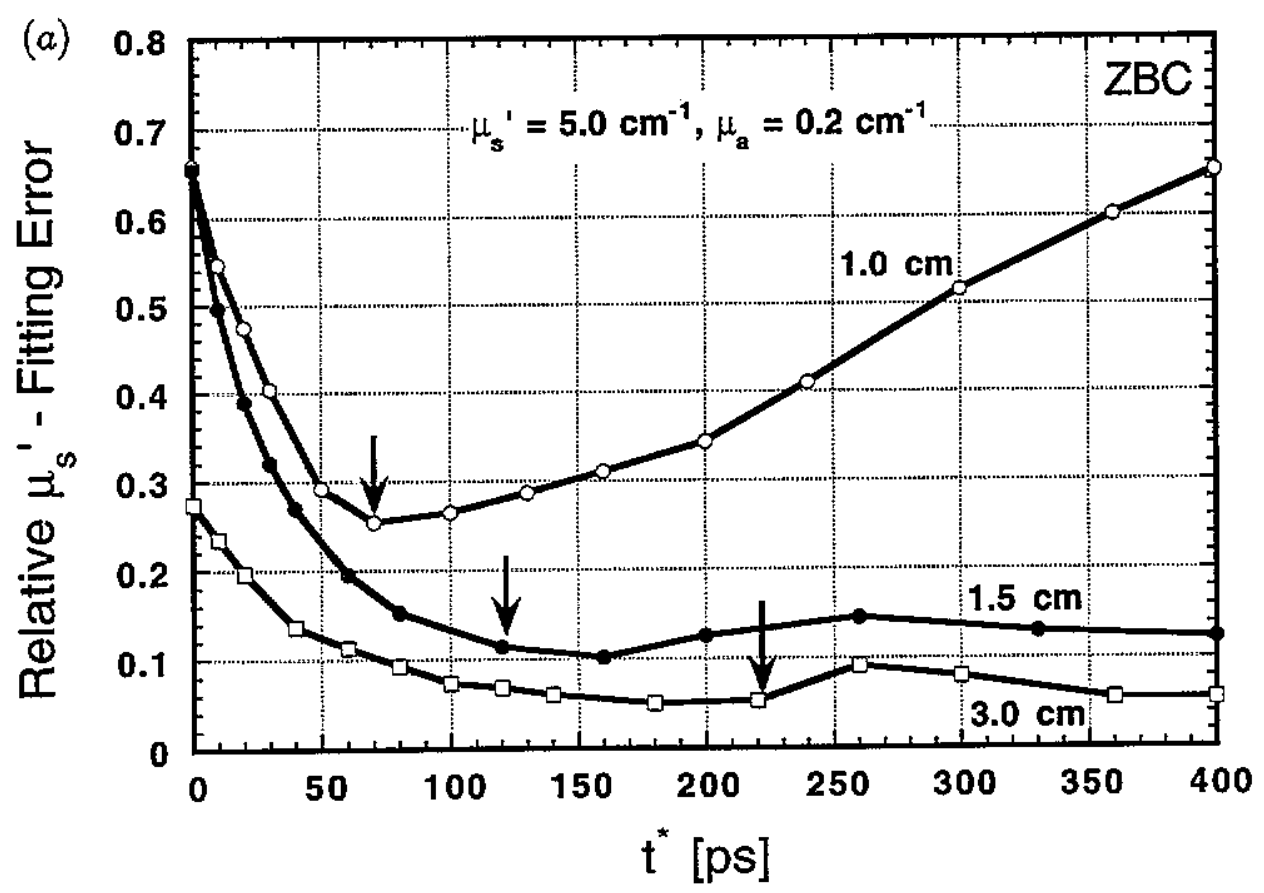

(b)

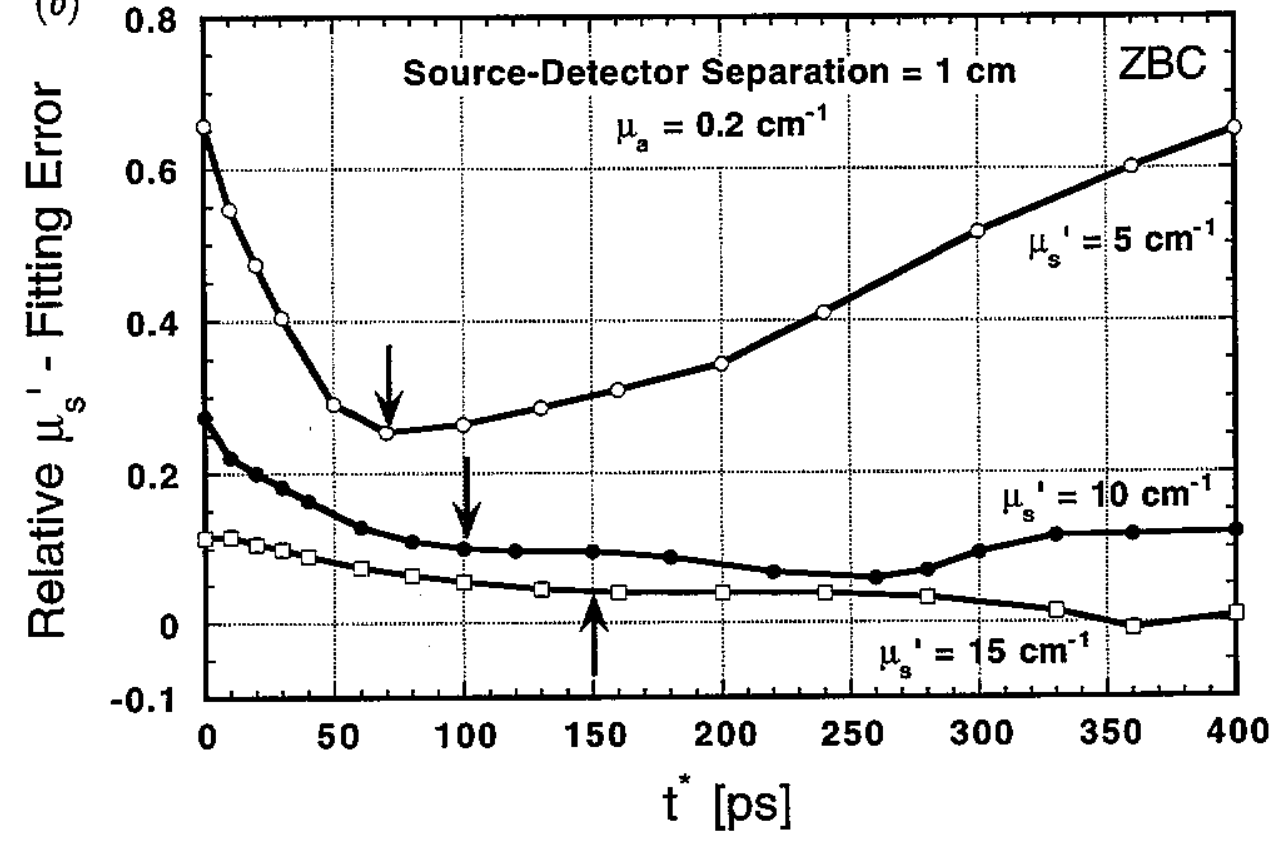

Figure 5. The relative error in the prediction of $\mu_{s}^{\prime}$, when $R_{Z B C}$ is used for fitting different parts of the reflectance data, indicated by the offset time $t^{*}$ : (a) the three curves correspond to three different source-detector separations; (b) the three curves correspond to three different $\mu_{s}^{\prime}$ with a source-detector separation of $1 \mathrm{~cm}$ in all cases. The arrows indicate data sets where only data points $t \geqslant t_{\max }$ are admitted for the fitting. 


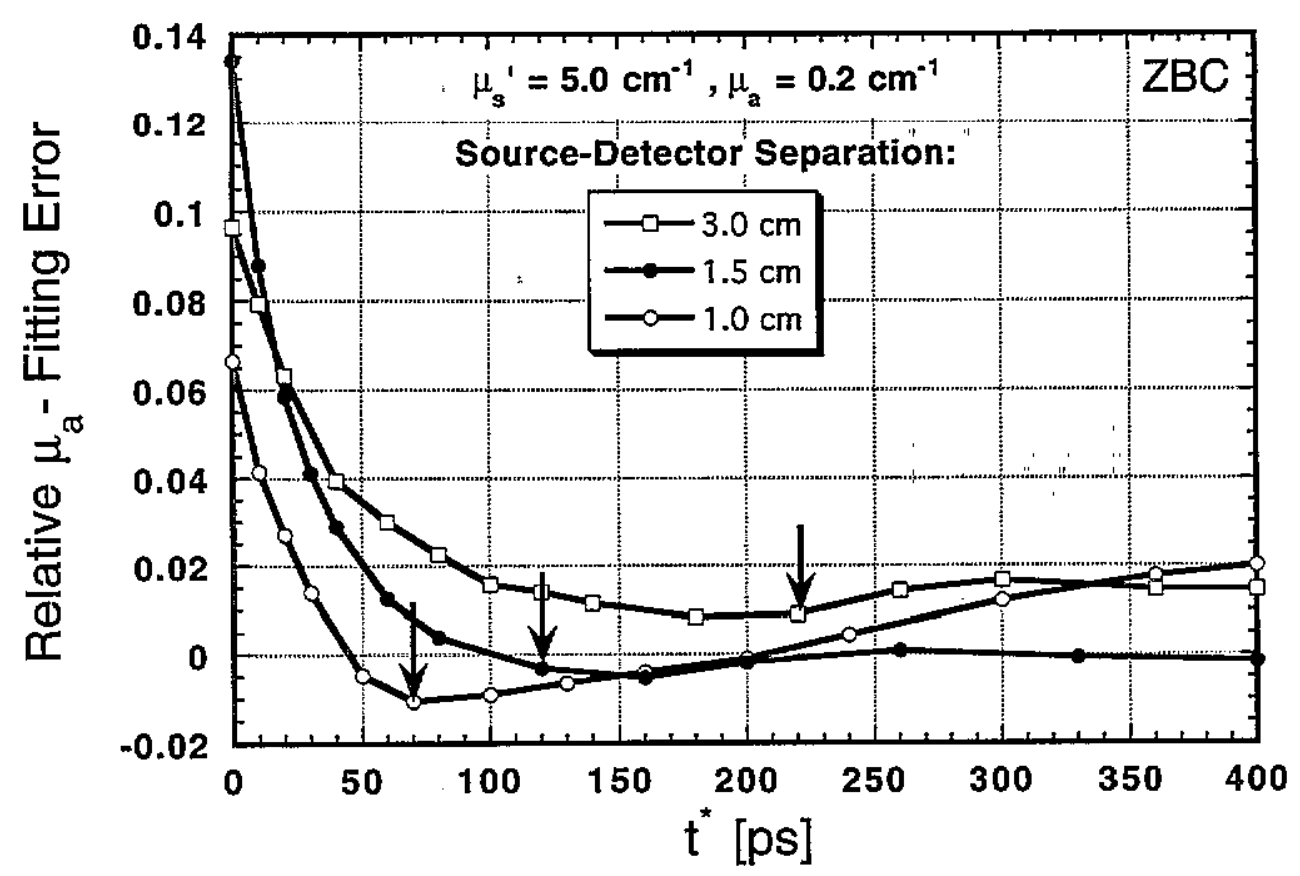

Figure 6. Relative error in the prediction of $\mu_{a}$, when $R_{Z B C}$ is used for fitting different parts of the reflectance data, indicated by the offset time $t^{*}$. The three curves correspond to three different source-detector separations and the arrows indicate data sets where only data points $t \geqslant t_{\text {max }}$ are admitted for the fitting.

\subsection{Comparison of Monte Carlo simulations with diffusion theory with the EPB}

Beside Monte Carlo simulations and $R_{Z B C}$, figure 4 also shows the time-resolved reflectance $R_{E P B}(r, t)$ (equation (16)), which was derived from diffusion theory with the EPB. Surprisingly $R_{E P B}$ does not give a better description than $R_{Z B C}$. While $R_{Z B C}$ overestimates the reflectance at small source-detector separations and low scattering coefficients, $R_{E P B}$ underestimates the reflectance in these cases. Thus, it seems that the more complicated approach of an extrapolated boundary does not improve the accuracy where the diffusion theory with $\mathrm{ZBC}$ fails.

That is furthermore supported by figure 7 . Here the fitting error in the determination of $\mu_{s}^{\prime}$ using an algorithm based on $R_{E P B}$ is displayed as a function of $t^{*}$. The curves are similar to those shown in figure 5 for the case of $R_{Z B C}$. However, the negative errors indicate that $\mu_{s}^{\prime}$ is now underestimated. For large source-detector separation and large $\mu_{s}^{\prime}$, the algorithm based on $R_{E P B}$ gives about the same error as the method based on $R_{Z B C}$, only this time with a negative sign.

Figure 8 shows the results for the determination of $\mu_{a}$ when a fitting algorithm based on $R_{E P B}$ is used. Similar to the results shown in figure 6, using all data leads here to an overestimation of $\mu_{a}$ by approximately $8-15 \%$. Omitting some early data points gives excellent fits of $\mu_{a}$ even for small source-detector separations and low scattering coefficients. 


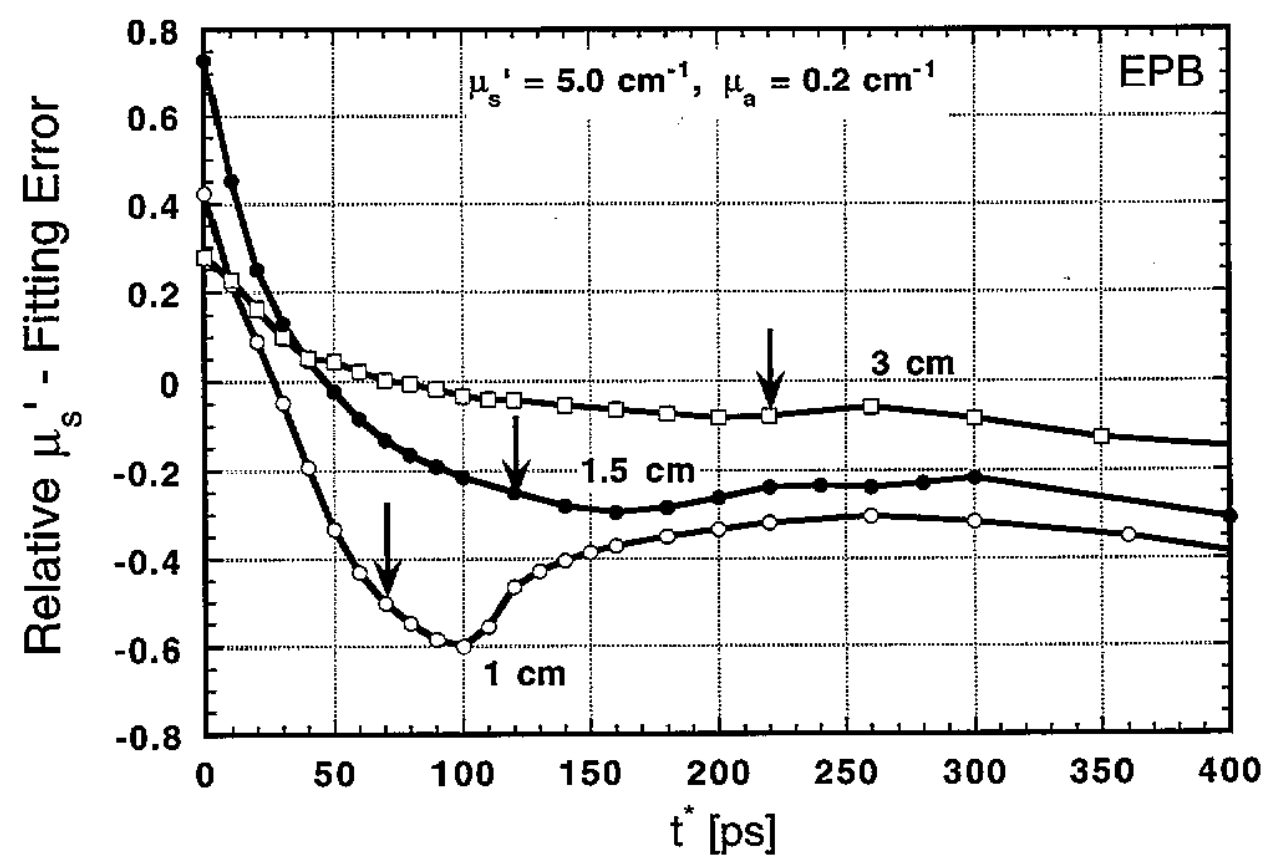

Figure 7. The relative error in the prediction of $\mu_{s}^{\prime}$, when $R_{E P B}$ is used for fitting different parts of the reflectance data, indicated by the offset time $t^{*}$. The three curves correspond to three different source-detector separations and the arrows indicate data sets where only data points $t \geqslant t_{\max }$ are used for the fitting.

\subsection{Comparison of Monte Carlo simulations with diffusion theory with the $P C B$}

As already pointed out in subsection 2.5 , the difference between $R_{E P B}$ and $R_{P C B}$ is smaller than $2 \%$. Depending on the number of photons and optical parameters used, the Monte Carlo simulations usually have a variance larger than $5 \%$. This remark also holds for actual measurements. Thus, for all practical purposes, no improvement is gained when $R_{P C B}$ is used. The appearance of the error function in equation (8) makes the implementation of a fast fitting routine rather difficult and thus not desirable.

\section{Monte Carlo simulations and experiments}

To test whether the reported results can be applied to actual time-resolved measurements, we also compared Monte Carlo simulations with experiments on tissue phantoms with known optical properties.

In figure 9, the experimental set-up for measuring the time-resolved reflectance on tissues or tissue phantoms is shown. The method of time-correlated photon counting was applied (O'Connor and Phillips 1984). As light sources laser diodes were used that were driven by a picosecond light pulser (PLP-02, Hamamatsu Photonics KK, Hamamatsu, Japan). Light pulses with a duration of $10-50 \mathrm{ps}$ (full width at half maximum) were emitted at a repetition rate of $10 \mathrm{MHz}$. The peak power reached $\sim 100 \mathrm{~mW}$ for the wavelengths of $780 \mathrm{~nm}$ and $830 \mathrm{~nm}$. The light was guided through a $200 \mu \mathrm{m}$ fibre to the tissue or tissue model. The scattered photons were collected at a distance $d$ and guided through a fibre bundle of 


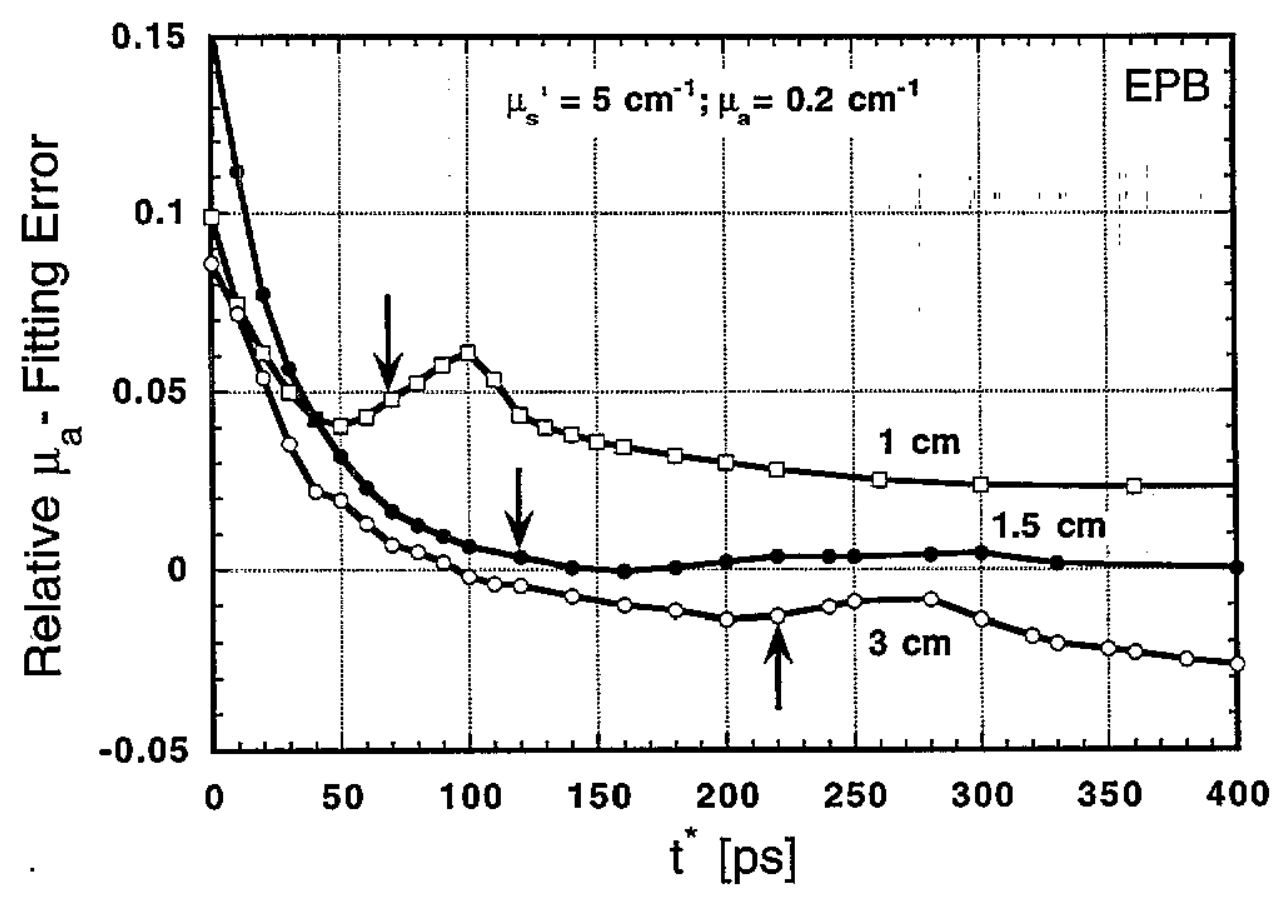

Figure 8. The relative error in the prediction of $\mu_{a}$, when $R_{E P B}$ is used for fitting different parts of the reflectance data, indicated by the offset time $t^{*}$. The three curves correspond to three different source-detector separations and the arrows indicate data sets where only data points $t \geqslant t_{\max }$ are used for the fitting.

$3 \mathrm{~mm}$ diameter to a microchannel plate-photomultiplier (MCP-PMT; R1564, Hamamatsu Photonics KK, Hamamatsu, Japan). Both input and collection fibres were fixed on a mount to assure constant spacing during a measurement.

The MCP-PMT signals were input to a constant fraction discriminator (CFD; TC454, Tennelec/Nucleus, Oak Ridge, TN, USA), via an amplifier and an attenuator. The CFD output was fed to a time to amplitude converter (TAC; 467, EG\&G Ortec, Oak Ridge, TN, USA), as a 'start counting' signal. The TAC outputs were counted as discrete events by a pulse height analyser (PHA; E-552, 562, 563, NAIG, Tokyo, Japan) and accumulated until the peak count reached 100000 . Then the time-response curve obtained was stored in a personal computer. The instrument function was measured after each measurement on the tissue phantoms by putting the detector and source fibres directly against each other. However, to prevent saturation of the MCP-PMT, neutral density filters were placed between the source and detector fibre ends. The instrument function was used to deconvolute the results from the phantom measurements yielding the corrected reflectance $R(r, t)$, which was compared with the Monte Carlo simulations.

The tissue phantoms were composed of polystyrene microspheres of $579 \mathrm{~nm} \pm 10 \mathrm{~nm}$ that served as the scatterers and india ink (Higgins, Faber-Castell Corporation, Lewisburg, TN, USA), that acted as the absorber. Solutions of different concentrations were contained in a cylindrical vessel with a radius of $6 \mathrm{~cm}$ and a depth of $10 \mathrm{~cm}$. The scattering coefficient of the media was calculated with Mie theory given the size, diffraction index, and concentration of the polystyrene spheres. The absorption coefficient was determined through calibration measurements of different concentrations of india ink in water. 


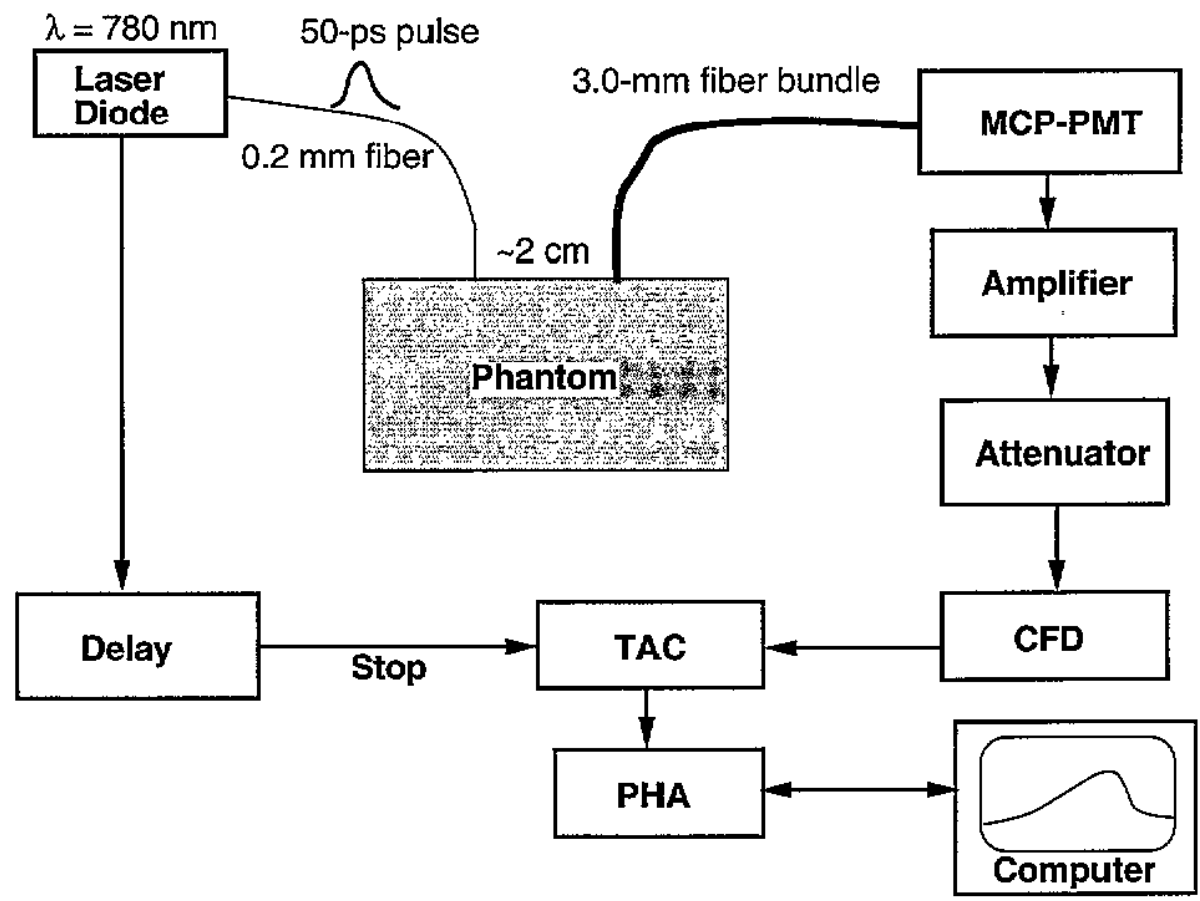

Figure 9. A block diagram of the experimental set-up for time-correlated single-photon counting (MCP-PMT, microchannel plate-photomultiplier; CFD, constant fraction discriminator; TAC, time to amplitude converter; PHA, pulse height analyser).

These theoretical values for $\mu_{s}^{s}(\mathrm{th})$ and $\mu_{a}$ (th) were used as optical parameters for a first Monte Carlo simulation. Subsequent Monte Carlo simulations were performed to minimize the error between experimental and simulation results. The Monte Carlo simulation yielding the best fit gives $\mu_{s}^{\prime}(\mathrm{MC})$ and $\mu_{a}(\mathrm{MC})$. We found that the difference between $\mu_{s}^{\prime}(\mathrm{th}), \mu_{a}(\mathrm{th})$ and $\mu_{s}^{\prime}(\mathrm{MC}), \mu_{a}(\mathrm{MC})$ was in all cases smaller than $10 \%$. The good agreement between Monte Carlo simulations and the experimental data (figure 10) confirms earlier reports from other groups finding that Monte Carlo simulations are an appropriate method to simulate photon migration in tissue (Flock et al 1989). That implies that the conclusions drawn from the comparison of diffusion theory and Monte Carlo simulations in the previous chapters also apply to experimental data.

\section{Discussion}

The question to be asked is, why does diffusion theory with the EPB give only negligible improvement compared to diffusion theory with the ZBC? Our findings suggest that the differences between time-resolved diffusion theory and Monte Carlo simulations and experiments cannot be resolved by choosing certain boundary conditions. The time-resolved diffusion theory has some inherent flaws that lead to an inaccurate description of the photon transport process in scattering media at early times.

The first source of error is given by the fact that diffusion theory is acausal (Kaltenbach and Kaschke 1993). The speed of propagation is infinite, meaning that any change in the fluence at any point in the medium has an immediate effect everywhere. This truly violates the physical situation that the speed of light is finite. After a pulse is launched into the 


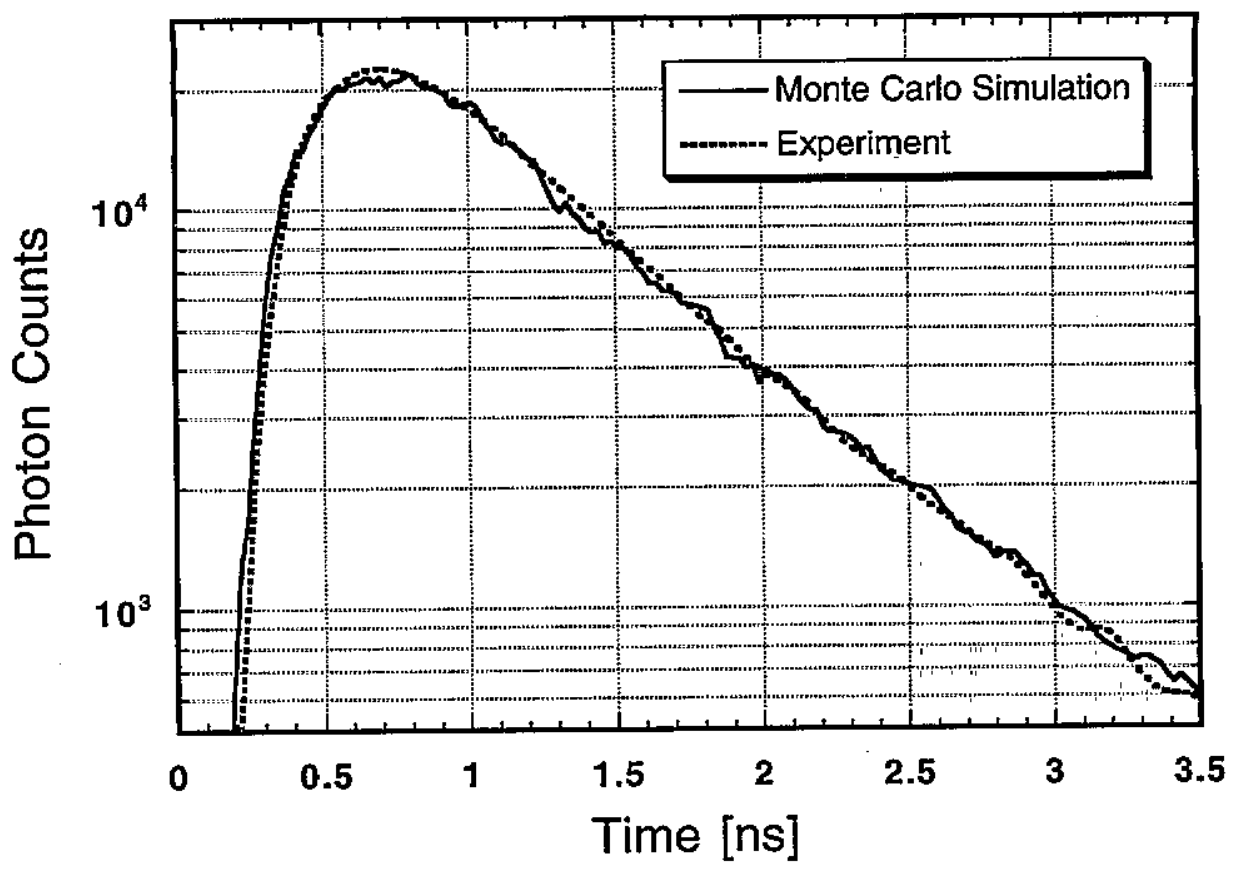

Figure 10. A comparison of Monte Carlo simulation and experiment. The source-detector separation is $2 \mathrm{~cm}, \mu_{a}=0.0273 \mathrm{~cm}^{-1}$, and $\mu_{s}^{\prime}=13.0 \mathrm{~cm}^{-1}$.

tissue, it takes a finite amount of time for the first photons to reach a detector. This problem is not resolved just by choosing different boundary conditions as figure 4 shows.

Second, in the derivation of the diffusion equation and its boundary conditions from the transport equation one has to assume that the scattering is isotropic (i.e. pointlike scatterers). On the other hand, in biological tissues, tissue phantoms, and our Monte Carlo simulations, the scattering is highly anisotropic. It is well known that diffusion theory can still be applied to anisotropic media if the scattering mean free path $1 / \mu_{s}$ is replaced by an effective scattering mean free path $1 /\left[(1-g) \mu_{s}\right]$ (Ishimaru 1978b), as we have done throughout this study. Because typically $g>0.9$ in biological tissues, the effective scattering mean free path is at least 10 times larger than the scattering mean free path. This means that at least 10 scattering events have to occur before a photon has lost its initial directionality and can be considered diffuse. In other words, enough time has to elapse to allow enough scattering events to occur that the photons become diffuse and diffusion theory can be applied successfully. The choice of the boundary condition does not influence this requirement.

\section{Summary}

In this work the formulas found from diffusion theory under three commonly used boundary conditions (zero, extrapolated, and partial current boundary conditions) were compared with each other, with results found from time-resolved Monte Carlo simulations and with measurements on phantom tissues. It is found that time-resolved diffusion theory with the extrapolated boundary condition (EPB) and diffusion theory with the partial current boundary condition $(\mathrm{PCB}$ ) predict almost the same reflectance for a given set of optical properties and source-detector separation. On the other hand, diffusion theory with the zero boundary condition (ZBC) underestimates the amplitude of the signal and displays sharper 
maxima.

All three diffusion theory approaches fail to accurately predict the results obtained from Monte Carlo sirnulations and experiments at early times. This leads to errors in the determination of the scattering coefficient, while the absorption coefficient can be extracted from given data sets with high accuracy. A quantification of the errors in the determination of optical properties shows that it is sufficient to use simple linear fitting algorithms based on diffusion theory with the $\mathrm{ZBC}$. Disregarding data before the maximum reflectance is reached leads to a minimal error in the prediction of $\mu_{s}^{\prime}$ and $\mu_{a}$. The more complex nonlinear fitting algorithms, based on the EPB or PCB, yield only negligible improvements in the extraction of $\mu_{s}^{\prime}$ and $\mu_{a}$.

Possible reasons for the failure of the EPB and PCB to yield a more accurate description of the actual photon transport are the acausal character of the diffusion theory and the fact that diffusion theory is derived under the assumption of isotropic scatterers.

\section{Acknowledgments}

The authors would like to thank Dr H Liu and Professor B Chance from the Department of Biochemistry and Biophysics at the University of Pennsylvania for support in the experimental set-up and the careful review of the paper. Furthermore the help of D Boas with the calculation of the scattering coefficient of the polystyrene sphere solutions is appreciated.

This work was supported by the Robert $A$ Welch Foundation, the Department of Energy, and NIH (R29-HL45045).

\section{Appendix. Derivations of $R_{E P B}\left(r, t, \mu_{a}, \mu_{s}^{\prime}\right)$}

Equation (16) can be written as

$$
R_{E P B}(r, t)=\gamma t^{-5 / 2} \exp \left(-\mu_{a} c t\right)\left(z_{0} \exp \left(h_{z_{0}}\right)+z_{p} \exp \left(h_{z_{p}}\right)\right)
$$

with

$$
\begin{aligned}
& h_{z_{0}}=-\left(z_{0}^{2}+r^{2}\right) / 4 D c t \\
& h_{z_{p}}=-\left(z_{p}^{2}+r^{2}\right) / 4 D c t .
\end{aligned}
$$

The amplitude $\gamma$ is an independent fitting parameter and thus we ignore the dependence on $\mu_{a}$ and $\mu_{s}^{\prime}$. Since $\mu_{s}^{\prime} \gg \mu_{a}$ for all cases considered in this work, we approximate $z_{0}=\left(\mu_{a}+\mu_{s}^{\prime}\right)^{-1} \approx\left(\mu_{s}^{\prime}\right)^{-1}$. While the derivation of equation (A1) with respect to $\gamma$ is obvious, the derivations with respect to $\mu_{a}$ and $\mu_{s}^{\prime}$ are given by

$$
\begin{aligned}
\left(\mathrm{d} / \mathrm{d} \mu_{a}^{\prime}\right) R_{E P B}(r, t) & =\gamma t^{-5 / 2} \exp \left(-\mu_{a} c t\right) \\
& \times\left(-c t+z_{0} \exp \left(h_{z_{0}}\right) \mathrm{d} h_{z_{0}} / \mathrm{d} \mu_{a}+\left(\mathrm{d} z_{p} / \mathrm{d} \mu_{a}\right) \exp \left(h_{z_{p}}\right)+z_{p} \exp \left(h_{z_{p}}\right) \mathrm{d} h_{z_{p}} / \mathrm{d} \mu_{a}\right)
\end{aligned}
$$

$$
\begin{gathered}
\left(\mathrm{d} / \mathrm{d} \mu_{s}^{\prime}\right) R_{E P B}(r, t)=\gamma t^{-5 / 2} \exp \left(-\mu_{a} c t\right)\left(\left(\mathrm{d} z_{0} / \mathrm{d} \mu_{s}^{\prime}\right) \exp \left(h_{z_{0}}\right)+z_{0} \exp \left(h_{z_{0}}\right)\left(\mathrm{d} h_{z_{0}} / \mathrm{d} \mu_{s}^{\prime}\right)\right. \\
\left.+\left(\mathrm{d} z_{p} / \mathrm{d} \mu_{s}^{\prime}\right) \exp \left(h_{z_{p}}\right)+z_{p} \exp \left(h_{z_{p}}\right)\left(\mathrm{d} h_{z_{p}} / \mathrm{d} \mu_{s}^{\prime}\right)\right)
\end{gathered}
$$

with

$$
\begin{aligned}
& \mathrm{d} z_{0} / \mathrm{d} \mu_{s}^{\prime}=-z_{0}^{2} \\
& \mathrm{~d} z_{p} / \mathrm{d} \mu_{s}^{\prime}=-z_{0}^{2}-4 A / 3\left(\mu_{a}+\mu_{s}^{\prime}\right)^{2} \\
& \mathrm{~d} z_{p} / \mathrm{d} \mu_{a}=-4 A / 3\left(\mu_{a}+\mu_{s}^{\prime}\right)^{2} \\
& \mathrm{~d} h_{z_{0}} / \mathrm{d} \mu_{s}^{\prime}=(-3 / 4 c t)\left(-z_{0}^{2}\left(2 \mu_{a}+1\right)+r^{2}\right)
\end{aligned}
$$




$$
\begin{aligned}
& \mathrm{d} h_{z_{p}} / \mathrm{d} \mu_{s}^{\prime}=(-3 / 4 c t)\left(-z_{0}^{2}\left(2 \mu_{a}+1+8 A / 3\right)+r^{2}-16 A^{2} / 9\left(\mu_{a}+\mu_{s}^{\prime}\right)^{2}\right) \\
& \mathrm{d} h_{z_{0}} / \mathrm{d} \mu_{a}=(-3 / 4 c t)\left(z_{0}^{2}+r^{2}\right) \\
& \mathrm{d} h_{z_{p}} / \mathrm{d} \mu_{a}=(-3 / 4 c t)\left(z_{0}^{2}+r^{2}-16 A^{2} / 9\left(\mu_{a}+\mu_{s}^{\prime}\right)^{2}\right) .
\end{aligned}
$$

The Levenberg-Marquardt nonlinear fitting that is used to fit $R_{E P B}$ to the Monte Carlo simulations algorithm requires the knowledge of these derivations.

\section{References}

Abramowitz M and Stegun I A 1972 Handbook of Mathematical Functions (New York: Dover)

Arridge S R, Cope M and Delpy D T 1992 The theoretical basis for the determination of optical pathiengths in tissue: temporal and frequency analysis Phys. Med. Biol. $371531-60$

Avraham D B, Taitelbaum $\mathrm{H}$ and Weiss $\mathrm{G} \mathrm{H} 1991$ Boundary conditions for a model of photon migration in a turbid medium Lasers Life Sct. 4 29-36

Benaron D A, Benitz W E, Ariagno R L and Stevenson D K 1992 Noninvasive methods for estimating in vivo oxygenation Clin, Pediatr. 31 258-73

Briesmeister J F (ed) 1986 MCNP-A General Monte Carlo Code for Neutron Photon Transport Version 3A, LA-7396-Manual (Los Alamos, NM: Los Alamos National Laboratory)

Carslaw H S and Jaeger J C 1959 Conduction of Heat in Solids (London: Oxford University Press)

Case K M and Zweifel P F 1967 Linear Transport Theory (Reading, MA: Addison-Wesley)

Chance B et al 1988 Comparison of time-resolved and -unresolved measurements of deoxyhemoglobin in brain Proc. Natl Acad. Sci. USA 85 4971-5

Deckelbaum IL I 1994 Cardiovascular application of laser technology Lasers Surg. Med. 15 315-41

Duderstadt J J and Hamilton L J 1976 Nuclear Reactor Analysis (New York: Wiley) pp 140-4

Eppstein J and Bursell S E 1992 Non-invasive detection of diabetes mellitus Proc. SPIE 1641 217-26

Farrell T J, Patterson M S and Wilson B C 1992 A diffusion theory model of spatially resolved, steady state diffuse reflectance for the non-invasive determination of tissue optical properties in vivo Med. Phys. 19 879-88

Flock S T, Wilson B C and Patterson M 1989 Monte Carlo modeling of light propagation in highly scattering tissues-II: comparison with measurements on phantoms IEEE Trans. Biomed. Eng, BME-36 1169-73

Glasstone S 1955 Principles of Nuclear Reactor Engineering (New York: Van Nostrand)

Groenhuis R A, Ferwerda A A and Bosch J J T 1983 Scattering and absorption of turbid materials determined from reflection measurements. 1: Theory Appl. Opt. 22 2456-62

Haskell R C, Svaasand L O, Tsay Tsong-Tseh, Feng Tu-Chen, McAdams M S and Tromberg B J 1994 Boundary conditions for the diffusion equation in radiative transfer $J$. Opt. Soc. Am. A $112727-41$

Ishimaru A 1978a Diffusion of a pulse in densely distributed scatterers J. Opt. Soc. Am. 68 1045-50

1978b Wave Propagation and Scattering in Random Media (New York: Academic)

Jacques S L 1989 Time-resolved reflectance spectroscopy in turbid tissue IEEE Trans. Biomed. Eng. BME-36 $1155-61$

Jacques S L, Gutsche A, Schwartz J, Wang L and Tittel F K 1993 Video reflectometry to extract optical properties of tissues in vivo Medical Optical Tomography: Functional Imaging and Monitoring (SPIE Institutes for Advanced Optical Technologies Series IS II) ed G Müller (Bellingham, WA: SPIE) pp 211-26

Jacques S L, Wang L H and Hielscher A H 1995 Time-resolved photon propagation in tissues Optical-Thermal Response of Laser-Irradiated Tissue ed A $J$ Welch and M van Gemert (New York: Plenum) pp 305-32

Kaltenbach J M and Kaschke M 1993 Frequency and time domain modelling of light transport in random media Medical Optical Tomography: Functional Imaging and Monitoring (SPIE Institutes for Advanced Optical Technologies Series IS 11) ed G Müller (Bellingham, WA: SPIE) pp 65-86

Keijzer M, Star M W and Storchi P R M 1988 Optical diffusion in layered media Appl. Opt. 27 1820-4

Kohl M, Essenpreis M and Cope M 1995 The influence of glucose concentration upon the transport of light in tissue-simulating phantoms Phys. Med. Biol. 40 1267-87

Moulton J D 1990 Diffusion modelling of picosecond laser pulse propagation in turbid media PhD Thesis McMaster University

Lux I and Koblinger L 1991 Monte Carlo Particle Transport Methods: Neutron and Photon Calculations (Boca Raton, FL: Chemical Rubber Company)

O'Connor D V and Phillips D 1984 Time-correlated Single Photon Counting (Orlando, FL: Academic)

Patterson M S, Chance B and Wilson B C 1989 Time resolved reflectance and transmittance for the noninvasive measurement of tissue optical properties Appl. Opt. $282331-6$ 
Press W H, Flannery B P, Teukolsky S A and Vetterling W T 1992 Numerical Recipes in C (New York: Cambridge University Press)

Ramanujam N, Mitchell M F, Mahadevan A, Warren S. Thomson S, Silva E and Richards-Kortum R 1994 In yivo diagnosis of cervical intraepithelial neoplasia using 337-nm-excited laser-induced fluorescence Proc. Natl Acad. Sci. USA 91 10193-7

Svaasand L O, Haskell D, Tromberg B and McAdams M 1993 Properties of photon density waves at boundaries Proc. SPIE 1888 214-26

Wang L H and Jacques S L 1995 Monte Carlo modeling of light transport Optical-Thermal Response of LaserIrradiated Tissue ed A J Welch and M van Gemeret (New York: Plenum) pp 73-100 (source code available through ftp anonymous @ laser.mda.uth.tmc.edu)

Wang L H, Jacques S L and Zheng L $1995 \mathrm{MCML}-$ Monte Carlo modeling of light transport in multilayered tissues Comput. Methods Programs Biomed. 47 131-46

Wilson B, Park Y, Hefetz Y, Patterson M, Madsen S and Jacques S L 1989 The potential of time-resolved reffectance measurements for noninvasive determination of tissue optical properties Proc. SPIE 1064 97-107 